\title{
UNIFORM CONSTANTS IN HAUSDORFF-YOUNG INEQUALITIES FOR THE CANTOR GROUP MODEL OF THE SCATTERING TRANSFORM
}

\author{
VJEKOSLAV KOVAČ
}

\begin{abstract}
Analogues of Hausdorff-Young inequalities for the Dirac scattering transform (a.k.a. $S U(1,1)$ nonlinear Fourier transform) were first established by Christ and Kiselev [1, 2]. Later Muscalu, Tao, and Thiele [5] raised a question if the constants can be chosen uniformly in $1 \leq p \leq 2$. Here we give a positive answer to that question when the Euclidean real line is replaced by its Cantor group model.
\end{abstract}

\section{INTRODUCTION}

The following context arises from the eigenfunction equation for the Dirac operator, after the natural ansatz is made, see [5]. Let $f: \mathbb{R} \rightarrow \mathbb{C}$ be a compactly supported integrable function. For any $\xi \in \mathbb{R}$ consider the initial value problem in the matrix form:

$$
\frac{\partial}{\partial x} G(x, \xi)=G(x, \xi) W(x, \xi), \quad G(-\infty, \xi)=\left[\begin{array}{ll}
1 & 0 \\
0 & 1
\end{array}\right],
$$

where

$$
G(x, \xi)=\left[\begin{array}{ll}
a(x, \xi) & \overline{b(x, \xi)} \\
b(x, \xi) & \overline{a(x, \xi)}
\end{array}\right], \quad W(x, \xi)=\left[\begin{array}{cc}
0 & \overline{f(x)} e^{-2 \pi i x \xi} \\
f(x) e^{2 \pi i x \xi} & 0
\end{array}\right] .
$$

The problem (1.1) has a unique solution with absolutely continuous functions $a(\cdot, \xi)$ and $b(\cdot, \xi)$ that satisfy the differential equation for a.e. $x \in \mathbb{R}$ and eventually become constant as $x \rightarrow-\infty$ or $x \rightarrow \infty$. The limit

$$
G(\infty, \xi)=\left[\begin{array}{ll}
a(\infty, \xi) & \overline{b(\infty, \xi)} \\
b(\infty, \xi) & \overline{a(\infty, \xi)}
\end{array}\right]=\lim _{x \rightarrow \infty}\left[\begin{array}{ll}
a(x, \xi) & \overline{b(x, \xi)} \\
b(x, \xi) & \overline{a(x, \xi)}
\end{array}\right]
$$

is a function in $\xi \in \mathbb{R}$, called the Dirac scattering transform of $f$. It is easy to see that all matrices $G(x, \xi)$ must belong to the Lie group

$$
\mathrm{SU}(1,1):=\left\{\left[\begin{array}{ll}
a & \bar{b} \\
b & \bar{a}
\end{array}\right]: a, b \in \mathbb{C},|a|^{2}-|b|^{2}=1\right\},
$$

and so $\xi \mapsto G(\infty, \xi)$ is indeed a function from $\mathbb{R}$ to $\mathrm{SU}(1,1)$. In analogy with the (linear) Fourier transform on $\mathbb{R}$, we also call it the $\mathrm{SU}(1,1)$ nonlinear Fourier transform of $f$, the term originating in 8 . We simply write $G(\xi), a(\xi), b(\xi)$ in place of $G(\infty, \xi), a(\infty, \xi), b(\infty, \xi)$. 
Using elementary contour integration one can show a "nonlinear analogue" of the Plancherel theorem:

$$
\left\|(2 \ln |a(\xi)|)^{1 / 2}\right\|_{\mathrm{L}_{\xi}^{2}(\mathbb{R})}=\|f\|_{\mathrm{L}^{2}(\mathbb{R})} .
$$

The first appearance of this identity (although in discrete setting) dates back to [9],[10]. From this equality it seems that $(\ln |a|)^{1 / 2}$ is the appropriate measure of size for matrices in $\mathrm{SU}(1,1)$, so in the spirit of classical Fourier analysis one can consider nonlinear analogues of Hausdorff-Young inequalities for $1 \leq p<2$ :

$$
\left\|(\ln |a(\xi)|)^{1 / 2}\right\|_{L_{\xi}^{q}(\mathbb{R})} \leq C_{p}\|f\|_{\mathrm{L}^{p}(\mathbb{R})},
$$

where $p$ and $q$ are conjugated exponents. Besides the trivial Riemann-Lebesgue type of estimate for $p=1$, one can show (1.3) for $1<p<2$, as is first done in [1], 2]. These papers also prove the maximal version of (1.3), i.e. Menshov-PaleyZygmund type inequality. Even stronger, variational estimates for $1 \leq p<2$ are shown recently in [7].

However, the truncation method from [1], 2] gives constants $C_{p}$ in (1.3) that blow up as $p \rightarrow 2-$. For that reason Muscalu, Tao, and Thiele raised the following conjecture in [5].

Conjecture 1.1. There exists a universal constant $C>0$ such that for any pair of conjugated exponents $1 \leq p \leq 2$ and $2 \leq q \leq \infty$ and every function $f$ as above one has

$$
\left\|(\ln |a(\xi)|)^{1 / 2}\right\|_{\mathrm{L}_{\xi}^{q}(\mathbb{R})} \leq C\|f\|_{\mathrm{L}^{p}(\mathbb{R})} .
$$

It is interesting to notice that, although we know that (1.3) holds in the endpoint case $p=2$, we still cannot conclude uniformity of $C_{p}$ for neighboring values of $p$. Such anomalies are not possible for linear operators due to the Riesz-Thorin interpolation theorem. However, our transformation $f \mapsto(\ln |a(\cdot)|)^{1 / 2}$ is truly nonlinear, and no standard interpolation result can be applied directly to prove the conjecture.

The goal of this paper is to prove Conjecture 1.1 in the case when the exponentials $e^{2 \pi i x \xi}$ in $W(x, \xi)$ are replaced by the character function $E_{d}(x, \xi)$ of the $d$-adic Cantor group model of the real line, rigorously defined in the next section. The method of the proof is a monotonicity argument over scales, which is typically a privilege of finitary group models. Such arguments are also sometimes called Bellman function proofs (see for instance [6]), as they require construction of an auxiliary function with certain monotonicity and convexity properties.

The main idea is taken from the "local proof" of the Cantor group model Plancherel theorem given in [5]. A new contribution is the construction of the modified "swapping function" $\beta_{d}$ that satisfies certain $\mathrm{L}^{p} \rightarrow \mathrm{L}^{q}$ estimates uniformly in $1 \leq p \leq 2$. In the proof we use linear Hausdorff-Young inequalities on $\mathbb{Z} / d \mathbb{Z}$, as a substitute for some cancellation identities in [5].

Let us remark that our qualitative assumption on $f$ is crucial in order to be able to define the scattering transform properly. If $f$ is merely in $\mathrm{L}^{p}(\mathbb{R})$ for $1 \leq p<2$ (but without compact support), then from maximal inequalities in [1], [2] it follows that the limit in (1.2) exists for a.e. $\xi \in \mathbb{R}$, but this is a rather nontrivial result. 
However, for $f \in \mathrm{L}^{2}(\mathbb{R})$ that is still an open problem, commonly known as the nonlinear Carleson theorem. Its Cantor group model variant is proven in [5]. One can still extend the definition of the scattering transform using density arguments, as in [8].

Acknowledgment. The author would like to thank his faculty advisor, Prof. Christoph Thiele, for suggesting the problem and for his help on improving the presentation.

\section{The MONOTONICITY ARGUMENT}

Fix an integer $d \geq 2$, and denote $\mathbb{Z}_{d}:=\mathbb{Z} / d \mathbb{Z}$. For any $x, \xi \in[0, \infty)$ that can be written uniquely $\sqrt{1}$ in base $d$ number system as $x=\sum_{n \in \mathbb{Z}} x_{n} d^{n}$ and $\xi=\sum_{n \in \mathbb{Z}} \xi_{n} d^{n}$, we define

$$
E_{d}(x, \xi):=e^{(2 \pi i / d) \sum_{n \in \mathbb{Z}} x_{n} \xi-1-n} .
$$

Then the $\mathrm{L}^{\infty}$ function $E_{d}:[0, \infty) \times[0, \infty) \rightarrow \mathrm{S}^{1}$ is called the Cantor group character function. To justify the name, we identify $[0, \infty)$ with a subgroup $\mathbb{A}_{d}$ of the infinite group product $\mathbb{Z}_{d}^{\mathbb{Z}}$ given by

$$
\begin{aligned}
\mathbb{A}_{d}:=\left\{\left(x_{n}\right)_{n \in \mathbb{Z}}:\right. & x_{n} \in \mathbb{Z}_{d} \text { for every } n \in \mathbb{Z}, \text { and there exists } \\
& \left.n_{0} \in \mathbb{Z} \text { such that } x_{n}=\mathbf{0} \text { for every } n \geq n_{0}\right\},
\end{aligned}
$$

via the identification $\mathbb{A}_{d} \rightarrow[0, \infty),\left(x_{n}\right)_{n \in \mathbb{Z}} \mapsto \sum_{n \in \mathbb{Z}} x_{n} d^{n}$. Then $E_{d}(\cdot, \cdot)$ realizes duality between $\mathbb{A}_{d}$ and its dual group $\hat{\mathbb{A}}_{d} \cong \mathbb{A}_{d}$.

For a compactly supported integrable function $f:[0, \infty) \rightarrow \mathbb{C}$ and $\xi \in[0, \infty)$ consider the initial value problem on $[0, \infty)$ :

$$
\frac{\partial}{\partial x} G(x, \xi)=G(x, \xi) W(x, \xi), \quad G(0, \xi)=\left[\begin{array}{ll}
1 & 0 \\
0 & 1
\end{array}\right]
$$

where

$$
G(x, \xi)=\left[\begin{array}{ll}
a(x, \xi) & \overline{b(x, \xi)} \\
b(x, \xi) & \overline{a(x, \xi)}
\end{array}\right], \quad W(x, \xi)=\left[\begin{array}{cc}
0 & \overline{f(x) E_{d}(x, \xi)} \\
f(x) E_{d}(x, \xi) & 0
\end{array}\right] .
$$

The limit

$$
G(\xi)=\left[\begin{array}{ll}
a(\xi) & \overline{b(\xi)} \\
b(\xi) & \overline{a(\xi)}
\end{array}\right]:=\lim _{x \rightarrow \infty}\left[\begin{array}{ll}
a(x, \xi) & \overline{b(x, \xi)} \\
b(x, \xi) & \overline{a(x, \xi)}
\end{array}\right]
$$

defines a function $\xi \mapsto G(\xi)$ from $[0, \infty)$ to $\mathrm{SU}(1,1)$, which we call the Cantor group model Dirac scattering transform of $f$. Dependence on $d$ is not notationally emphasized but is understood. If for some interval $I \subseteq[0, \infty)$ we replace $f$ by $f \mathbf{1}_{I}$, then we will denote the corresponding $G, a, b$ respectively by $G_{I}, a_{I}, b_{I}$.

The main result of the paper is the following theorem.

Theorem 2.1. For every integer $d \geq 2$ there exists a constant $C_{d}>0$ such that for any pair of conjugated exponents $1 \leq p \leq 2$ and $2 \leq q \leq \infty$ and every function $f$ as above one has

$$
\left\|(\ln |a(\xi)|)^{1 / 2}\right\|_{L_{\xi}^{q}(\mathbb{R})} \leq C_{d}\|f\|_{L^{p}(\mathbb{R})} .
$$

\footnotetext{
${ }^{1}$ Because of ambiguous base $d$ representation of some reals, the function $E_{d}$ is not well-defined on a set of measure zero. The same comment applies to the later identification of $\mathbb{A}_{d}$ with $[0, \infty)$.
} 
The proof is given below, with the main technical construction postponed until the next section. In the following exposition we need a couple of simple facts proved in [5].

Lemma 2.2 (from [5]). If I and $\omega$ are two d-adic intervald with $|I||\omega|=1$, then $\xi \mapsto\left|a_{I}(\xi)\right|$ and $\xi \mapsto\left|b_{I}(\xi)\right|$ are constant functions on $\omega$.

We will be working in the phase space $\mathbb{A}_{d} \times \hat{\mathbb{A}}_{d}$, which is identified with $[0, \infty) \times$ $[0, \infty)$. Tiles and multitiles are rectangles of the form $I \times \omega$ for two $d$-adic intervals $I, \omega$ satisfying $|I||\omega|=1$ and $|I||\omega|=d$ respectively. Every multitile $I \times \omega$ can be partitioned into $d$ tiles by subdividing either $I$ or $\omega$ into $d$ congruent $d$-adic intervals. Lemma 2.2 motivates us to define $G_{P}, a_{P}, b_{P}$ for any tile $P=I \times \omega$ simply as $G_{I}\left(\xi_{\omega}\right), a_{I}\left(\xi_{\omega}\right), b_{I}\left(\xi_{\omega}\right)$, where $\xi_{\omega}$ is the left endpoint of $\omega$.
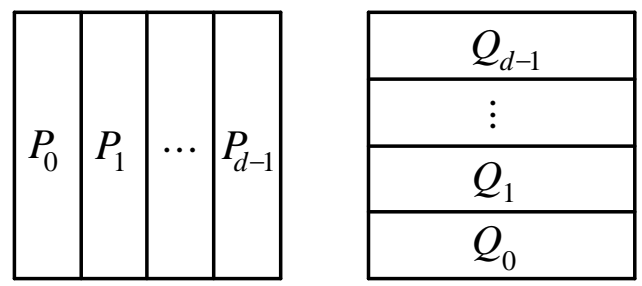

Figure 1. A multitile partitioned in two ways.

Lemma 2.3 (from [5]). Suppose that a multitile is divided horizontally into tiles $P_{0}, \ldots, P_{d-1}$, and vertically into tiles $Q_{0}, \ldots, Q_{d-1}$, as in Figure 1. Then

$$
\left[\begin{array}{cc}
a_{Q_{k}} & \overline{b_{Q_{k}}} \\
b_{Q_{k}} & \overline{a_{Q_{k}}}
\end{array}\right]=\prod_{j=0}^{d-1}\left[\begin{array}{cc}
a_{P_{j}} & \overline{b_{P_{j}}} e^{-2 \pi i j k / d} \\
b_{P_{j}} e^{2 \pi i j k / d} & \overline{a_{P_{j}}}
\end{array}\right]
$$

for $k=0,1, \ldots, d-1$. (The matrix product has to be taken in ascending order.)

This section concludes with the proof of Theorem 2.1, assuming that the following proposition holds.

Proposition 2.4. There exist a constant $C_{d}>0$ and a function $\beta_{d}:[0, \infty) \rightarrow$ $[0, \infty)$ such that for every $\left[\begin{array}{ll}a & \bar{b} \\ b & \bar{a}\end{array}\right] \in \mathrm{SU}(1,1)$

$$
C_{d}^{-1}(\ln |a|)^{1 / 2} \leq \beta_{d}(|b|) \leq C_{d}(\ln |a|)^{1 / 2},
$$

and whenever matrices $\left[\begin{array}{ll}a_{j} & \overline{b_{j}} \\ b_{j} & \overline{a_{j}}\end{array}\right],\left[\begin{array}{ll}A_{k} & \overline{B_{k}} \\ B_{k} & \overline{A_{k}}\end{array}\right] \in \mathrm{SU}(1,1), j, k=0,1, \ldots, d-1$ satisfy

$$
\left[\begin{array}{cc}
A_{k} & \overline{B_{k}} \\
B_{k} & \overline{A_{k}}
\end{array}\right]=\prod_{j=0}^{d-1}\left[\begin{array}{cc}
a_{j} & \overline{b_{j}} e^{-2 \pi i j k / d} \\
b_{j} e^{2 \pi i j k / d} & \overline{a_{j}}
\end{array}\right],
$$

then for any pair of conjugated exponents $1<p \leq 2$ and $2 \leq q<\infty$ one has

$$
\left(\frac{1}{d} \sum_{k=0}^{d-1} \beta_{d}\left(\left|B_{k}\right|\right)^{q}\right)^{\frac{1}{q}} \leq\left(\sum_{j=0}^{d-1} \beta_{d}\left(\left|b_{j}\right|\right)^{p}\right)^{\frac{1}{p}} .
$$

\footnotetext{
${ }^{2}$ These are intervals of the form $\left[d^{n} m, d^{n}(m+1)\right)$, for some $m, n \in \mathbb{Z}, m \geq 0$.
} 
This proposition is proved in the next section, by giving an explicit construction of $\beta_{d}$. The construction might seem a bit tedious, but we have to satisfy (2.3) with the exact constant at most 1 , since we will be repeatedly applying that inequality in the proof of Theorem 2.1. Iterating an inequality with a constant $C>1$ would not yield an estimate independent of the number of scales.

A consequence of Lemma 2.3 and (2.3) is that for $P_{0}, \ldots, P_{d-1}, Q_{0}, \ldots, Q_{d-1}$ as above we get

$$
\left(\frac{1}{d} \sum_{k=0}^{d-1} \beta_{d}\left(\left|b_{Q_{k}}\right|\right)^{q}\right)^{\frac{1}{q}} \leq\left(\sum_{j=0}^{d-1} \beta_{d}\left(\left|b_{P_{j}}\right|\right)^{p}\right)^{\frac{1}{p}} .
$$

Proof of Theorem 2.1 assuming Proposition 2.4.

We can consider $1<p \leq 2$, as for $p=1$ the estimate is an immediate consequence of Gronwall's inequality. Fix a positive integer $N$ (large enough) so that $f$ is supported in $\left[0, d^{N}\right)$. In all of the following we consider only those tiles $I \times \omega$ that are subsets of $\left[0, d^{N}\right) \times\left[0, d^{N}\right)$. For any $n \in \mathbb{Z},-N \leq n \leq N$ consider the following quantity:

$$
\mathcal{B}_{n}:=\left(\sum_{|I|=d^{n}}\left(d^{-n} \sum_{|\omega|=d^{-n}} \beta_{d}\left(\left|b_{I \times \omega}\right|\right)^{q}\right)^{\frac{p}{q}}\right)^{\frac{1}{p}} .
$$

In words, we consider all tiles $P$ of type $d^{n} \times d^{-n}$, then we take normalized $\ell^{q}$-norm of numbers $\beta_{d}\left(\left|b_{P}\right|\right)$ for all tiles in the same column, and finally we take $\ell^{p}$-norm of those numbers over all columns. Let us first prove that this quantity is decreasing in $n$.

$$
\mathcal{B}_{n+1}^{p}=\sum_{|I|=d^{n+1}}\left(d^{-n} \sum_{|\omega|=d^{-n}} d^{-1} \sum_{\substack{\omega^{\prime} \subseteq \omega \\\left|\omega^{\prime}\right|=d^{-n-1}}} \beta_{d}\left(\left|b_{I \times \omega^{\prime}}\right|\right)^{q}\right)^{\frac{p}{q}}
$$

using (2.4) for the multitile $I \times \omega$

$$
\leq \sum_{|I|=d^{n+1}}\left(d^{-n} \sum_{|\omega|=d^{-n}}\left(\sum_{\substack{I^{\prime} \subseteq I \\\left|I^{\prime}\right|=d^{n}}} \beta_{d}\left(\left|b_{I^{\prime} \times \omega}\right|\right)^{p}\right)^{\frac{q}{p}}\right)^{\frac{p}{q}}
$$

using Minkowski's inequality, since $q / p \geq 1$

$$
\leq \sum_{|I|=d^{n+1}} \sum_{\begin{array}{c}
I^{\prime} \subseteq I \\
\left|I^{\prime}\right|=d^{n}
\end{array}}\left(d^{-n} \sum_{|\omega|=d^{-n}} \beta_{d}\left(\left|b_{I^{\prime} \times \omega}\right|\right)^{q}\right)^{\frac{p}{q}}=\mathcal{B}_{n}^{p}
$$

Furthermore, when $n=-N$ we have:

$$
\begin{array}{r}
\mathcal{B}_{-N}=\left(\sum_{|I|=d^{-N}}\left(d^{N}\right)^{\frac{p}{q}} \beta_{d}\left(\left|b_{I \times\left[0, d^{N}\right)}\right|\right)^{p}\right)^{\frac{1}{p}} \leq C_{d}\left(d^{N}\right)^{\frac{1}{q}}\left(\sum_{|I|=d^{-N}}\left(\ln \left|a_{I}(0)\right|\right)^{\frac{p}{2}}\right)^{\frac{1}{p}} \\
\leq C_{d}\left(d^{N}\right)^{\frac{1}{q}}\left(\sum_{|I|=d^{-N}}\left\|f \mathbf{1}_{I}\right\|_{\mathrm{L}^{1}}^{p}\right)^{\frac{1}{p}} \leq C_{d}\|f\|_{\mathrm{L}^{p}} .
\end{array}
$$


Here we have applied the trivial $\mathrm{L}^{1}-\mathrm{L}^{\infty}$ estimate $\left(\ln \left|a_{I}(0)\right|\right)^{1 / 2} \leq\left\|f \mathbf{1}_{I}\right\|_{\mathrm{L}^{1}}$ and Hölder's inequality $\left\|f \mathbf{1}_{I}\right\|_{\mathrm{L}^{1}} \leq\left\|f \mathbf{1}_{I}\right\|_{\mathrm{L}^{p}}\left\|\mathbf{1}_{I}\right\|_{\mathrm{L}^{q}}$. On the other hand, for $n=N$ we have:

$$
\begin{array}{r}
\mathcal{B}_{N}=\left(d^{-N} \sum_{|\omega|=d^{-N}} \beta_{d}\left(\left|b_{\left[0, d^{N}\right) \times \omega}\right|\right)^{q}\right)^{\frac{1}{q}} \geq C_{d}^{-1}\left(d^{-N} \sum_{|\omega|=d^{-N}}\left(\ln \left|a\left(\xi_{\omega}\right)\right|\right)^{\frac{q}{2}}\right)^{\frac{1}{q}} \\
=C_{d}^{-1}\left(\int_{0}^{d^{N}}(\ln |a(\xi)|)^{\frac{q}{2}} d \xi\right)^{\frac{1}{q}} .
\end{array}
$$

Above $\xi_{\omega}$ denotes the left endpoint of $\omega$ and we have used that $\xi \mapsto|a(\xi)|$ is constant on intervals of length $d^{-N}$, by Lemma 2.2. From the monotonicity of $\left(\mathcal{B}_{n}\right)$ we conclude:

$$
\left(\int_{0}^{d^{N}}(\ln |a(\xi)|)^{\frac{q}{2}} d \xi\right)^{\frac{1}{q}} \leq C_{d} \mathcal{B}_{N} \leq C_{d} \mathcal{B}_{-N} \leq C_{d}^{2}\|f\|_{L^{p}}
$$

and by taking $\lim _{N \rightarrow \infty}$ we deduce the theorem.

\section{The SWAPPING INEQUALiTy}

This technical section is devoted to the proof of Proposition 2.4, An arbitrary function on $\mathbb{Z}_{d}$ can be presented as a complex $d$-tuple $\left(z_{0}, z_{1}, \ldots, z_{d-1}\right)$. Its Fourier transform is the $d$-tuple $\left(Z_{0}, Z_{1}, \ldots, Z_{d-1}\right)$ given by

$$
Z_{k}:=\sum_{j=0}^{d-1} z_{j} e^{2 \pi i j k / d}
$$

Lemma 3.1. For a pair of conjugated exponents $1<p \leq 2$ and $2 \leq q<\infty$ and $\left(z_{j}\right),\left(Z_{k}\right)$ as above, one has

$$
\left(\frac{1}{d} \sum_{k=0}^{d-1}\left|Z_{k}\right|^{q}\right)^{\frac{1}{q}} \leq\left(\sum_{j=0}^{d-1}\left|z_{j}\right|^{p}\right)^{\frac{1}{p}}
$$

Lemma 3.1 is a particular consequence of the general theory of the Fourier transform on locally compact abelian groups (see [3]). Indeed, one observes that the (non-stated) case $p=1$ is trivial from the triangle inequality, while for $p=2$ we indeed have an equality that follows from orthonormality of group characters. Intermediate cases are deduced by interpolating these two endpoint ones using the Riesz-Thorin theorem, since the transformation $\left(z_{j}\right) \mapsto\left(Z_{k}\right)$ is linear.

For any integer $d \geq 2$ let $t_{d}$ be the unique solution of the equation

$$
t e^{-t}=(2 d)^{-5} \sqrt{1+\operatorname{arsinh} t}
$$

that lies in $[0,1]$. One can easily see

$$
2^{-5} d^{-5}<t_{d}<2^{-4} d^{-5}
$$

and indeed $t_{d}=(2 d)^{-5}+\frac{3}{2}(2 d)^{-10}+\mathrm{O}\left(d^{-15}\right)$ as $d \rightarrow \infty$, but we do not need bounds on $t_{d}$ that are more precise than (3.1). 
Now we define $\beta_{d}:[0, \infty) \rightarrow[0, \infty)$ by the formula

$$
\beta_{d}(t):=\left\{\begin{array}{cl}
t e^{-t}, & \text { for } t \leq t_{d} \\
(2 d)^{-5} \sqrt{1+\operatorname{arsinh} t}, & \text { for } t>t_{d}
\end{array}\right.
$$

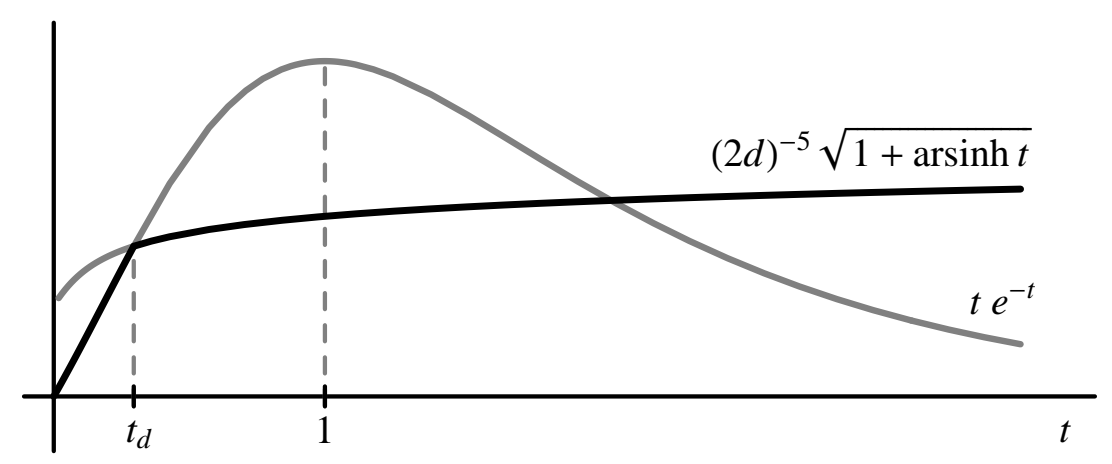

Figure 2. Graph of $\beta_{d}$. Not drawn to scale.

Using only basic calculus, one can easily establish the following properties of $\beta_{d}$ :

$$
\begin{aligned}
& 2^{-6} d^{-5} \sqrt{\ln \left(1+t^{2}\right)} \leq \beta_{d}(t) \leq 2 \sqrt{\ln \left(1+t^{2}\right)} \\
& \beta_{d}(t) \leq t e^{-t}, \quad \text { for } 0 \leq t \leq 1, \\
& \beta_{d}(t) \leq(2 d)^{-5} \sqrt{1+\operatorname{arsinh} t}, \quad \text { for any } t \geq 0 .
\end{aligned}
$$

Since (3.2) is exactly (2.1), it is enough to verify (2.3).

By performing matrix multiplication in (2.2), one can write $B_{k}$ explicitly as a sum of $2^{d-1}$ terms of the form

$$
\begin{aligned}
& \overline{a_{0}} \ldots \overline{a_{j_{1}-1}} b_{j_{1}} a_{j_{1}+1} \ldots a_{j_{2}-1} \overline{b_{j_{2}}} \overline{a_{j_{2}+1}} \ldots \overline{a_{j_{3}-1}} b_{j_{3}} a_{j_{3}+1} \ldots \\
& \ldots a_{j_{2 r}-1} \overline{b_{j_{2 r}}} \overline{a_{j_{2 r}+1}} \ldots \overline{a_{j_{2+1}-1}} b_{j_{2 r+1}} a_{j_{2 r+1}+1} \ldots a_{d-1} \cdot e^{(2 \pi i k / d)\left(j_{1}-j_{2}+j_{3}-\ldots-j_{2 r}+j_{2 r+1}\right)}
\end{aligned}
$$

where the summation is taken over all integers $0 \leq r \leq\left\lfloor\frac{d-1}{2}\right\rfloor$ and over all possible choices of indices $0 \leq j_{1}<j_{2}<\ldots<j_{2 r+1} \leq d-1$. In particular, observe that each term contains an odd number of $b$ 's. Terms that contain exactly one of the $b$ 's could be called linear terms, and so the "linear part" of $B_{k}$ is

$$
B_{k}^{\prime}:=\sum_{j=0}^{d-1} \overline{a_{0} a_{1} \ldots a_{j-1}} b_{j} a_{j+1} \ldots a_{d-1} e^{2 \pi i j k / d}, \quad \text { for } k=0,1, \ldots, d-1 .
$$

Other terms in $B_{k}$ are called nonlinear terms. Observe that Lemma 3.1 gives

$$
\left(\frac{1}{d} \sum_{k=0}^{d-1}\left|B_{k}^{\prime}\right|^{q}\right)^{\frac{1}{q}} \leq\left(\sum_{j=0}^{d-1}\left|b_{j}^{\prime}\right|^{p}\right)^{\frac{1}{p}}
$$


where $b_{j}^{\prime}:=\overline{a_{0} \ldots a_{j-1}} b_{j} a_{j+1} \ldots a_{d-1}$. In the case when some $\left|b_{m}\right|$ is "large" and all other $\left|b_{j}\right|, j \neq m$ are "small" we find the following variant more useful:

$$
\begin{aligned}
& B_{k}^{\prime \prime}:=\sum_{j=0}^{m-1} \overline{c_{0} \ldots c_{j-1}} b_{j} c_{j+1} \ldots c_{m-1} a_{m} c_{m+1} \ldots c_{d-1} e^{2 \pi i j k / d} \\
& +\overline{c_{0} \ldots c_{m-1}} b_{m} c_{m+1} \ldots c_{d-1} e^{2 \pi i m k / d} \\
& +\sum_{j=m+1}^{d-1} \frac{}{c_{0} \ldots c_{m-1} a_{m} c_{m+1} \ldots c_{j-1}} b_{j} c_{j+1} \ldots c_{d-1} e^{2 \pi i j k / d},
\end{aligned}
$$

where we have denoted $c_{j}:=a_{j} /\left|a_{j}\right|$. This time Lemma 3.1 implies

$$
\left(\frac{1}{d} \sum_{k=0}^{d-1}\left|B_{k}^{\prime \prime}\right|^{q}\right)^{\frac{1}{q}} \leq\left(\left|b_{m}\right|^{p}+\left|a_{m}\right|^{p} \sum_{j \neq m}\left|b_{j}\right|^{p}\right)^{\frac{1}{p}} .
$$

The proof strategy is to compare $B_{k}$ to $B_{k}^{\prime}$ or $B_{k}^{\prime \prime}$ by estimating nonlinear terms, and then use inequalities (3.5) or (3.6). As we will soon see, $\beta_{d}$ is carefully chosen so that it compensates for the perturbation caused by nonlinear terms.

Choose indices $m, m^{*} \in\{0, \ldots, d-1\}$ such that $\left|b_{m}\right|$ is the largest among the numbers $\left|b_{j}\right|$, and $\left|b_{m^{*}}\right|$ is the largest among the numbers $\left|b_{j}\right| ; j \neq m$, i.e. the second largest among $\left|b_{j}\right|$. We distinguish the following three cases.

Case 1. $\left|b_{j}\right| \leq t_{d}$ for every $j$.

Recall that $\left|a_{j}\right|^{2}-\left|b_{j}\right|^{2}=1$, which implies $\left|a_{j}\right| \leq 1+\left|b_{j}\right| \leq 1+t_{d}$. We begin with a rough estimate obtained using (3.1):

$$
\left|B_{k}\right| \leq \sum_{j=0}^{d-1}\left|b_{j}\right|\left(\prod_{l \neq j}\left(\left|a_{l}\right|+\left|b_{l}\right|\right)\right) \leq d t_{d}\left(1+2 t_{d}\right)^{d-1} \leq 2^{-3} d^{-4}
$$

which guarantees $\left|B_{k}\right| \leq 1$, and thus $\beta_{d}\left(\left|B_{k}\right|\right) \leq\left|B_{k}\right| e^{-\left|B_{k}\right|}$ by (3.3). Therefore it is enough to prove

$$
\left(\frac{1}{d} \sum_{k=0}^{d-1}\left|B_{k}\right|^{q} e^{-q\left|B_{k}\right|}\right)^{\frac{1}{q}} \leq\left(\sum_{j=0}^{d-1}\left|b_{j}\right|^{p} e^{-p\left|b_{j}\right|}\right)^{\frac{1}{p}} .
$$

Lemma 3.2.

$$
\begin{aligned}
& \left(\frac{1}{d} \sum_{k=0}^{d-1}\left|B_{k}\right|^{q} e^{-q\left|B_{k}\right|}\right)^{\frac{1}{q}} \leq\left(\left.\frac{1}{d} \sum_{k=0}^{d-1}\left|B_{k}^{\prime}\right|\right|^{q} e^{-q\left|B_{k}^{\prime}\right|}\right)^{\frac{1}{q}}+2^{-3} d^{-2}\left|b_{m^{*}}\right|^{2} \\
& \left(\frac{1}{d} \sum_{k=0}^{d-1}\left|B_{k}^{\prime}\right|^{q} e^{\left.-q\left|B_{k}^{\prime}\right|\right)^{\frac{1}{q}}} \leq\left\|b^{\prime}\right\|_{\ell^{p}} e^{-\left\|b^{\prime}\right\|_{\ell p}}\right. \\
& \left\|b^{\prime}\right\|_{\ell^{p}} e^{-\left\|b^{\prime}\right\|_{\ell p}} \leq\left(\sum_{j=0}^{d-1}\left|b_{j}\right|^{p} e^{-p\left|b_{j}\right|}\right)^{\frac{1}{p}}-2^{-3} d^{-2}\left|b_{m^{*}}\right|^{2}
\end{aligned}
$$

Here we have denoted $\left\|b^{\prime}\right\|_{\ell^{p}}:=\left(\sum_{j=0}^{d-1}\left|b_{j}^{\prime}\right|^{p}\right)^{1 / p}$. 
The desired inequality is obtained simply by adding the three estimates above.

Proof of Lemma 3.2. We start by showing (3.7). Since $B_{k}-B_{k}^{\prime}$ contains only nonlinear terms and these have at least $3 b$ 's, we have the following error estimate:

$$
\begin{aligned}
\left|B_{k}-B_{k}^{\prime}\right| & \leq \sum_{j_{1}<j_{2}<j_{3}}\left|b_{j_{1}}\right|\left|b_{j_{2}}\right|\left|b_{j_{3}}\right|\left(\prod_{l \neq j_{1}, j_{2}, j_{3}}\left(\left|a_{l}\right|+\left|b_{l}\right|\right)\right) \\
& \leq d^{3}\left|b_{m}\right|\left|b_{m^{*}}\right|^{2}\left(1+2 t_{d}\right)^{d-3} \leq 2^{-3} d^{-2}\left|b_{m^{*}}\right|^{2} .
\end{aligned}
$$

(For $d=2$ this difference is 0.) By the mean value theorem for $t e^{-t}$ :

$$
|| B_{k}\left|e^{-\left|B_{k}\right|}-\right| B_{k}^{\prime}\left|e^{-\left|B_{k}^{\prime}\right|}\right| \leq 2^{-3} d^{-2}\left|b_{m^{*}}\right|^{2},
$$

and it remains to use Minkowski's inequality.

In order to prove (3.8) we consider the function $\varphi(t):=t e^{-q t^{1 / q}}$, which is increasing and concave on $[0,1]$ since:

$$
\varphi^{\prime}(t)=e^{-q t^{1 / q}}\left(1-t^{1 / q}\right)>0, \quad \varphi^{\prime \prime}(t)=\frac{1}{q} e^{-q t^{1 / q}} t^{1 / q-1}\left(-1-q+q t^{1 / q}\right)<0,
$$

for $0<t<1$. Now (3.8) follows using Jensen's inequality and (3.5):

$$
\frac{1}{d} \sum_{k=0}^{d-1} \varphi\left(\left|B_{k}^{\prime}\right|^{q}\right) \leq \varphi\left(\frac{1}{d} \sum_{k=0}^{d-1}\left|B_{k}^{\prime}\right|^{q}\right) \leq \varphi\left(\left(\sum_{j=0}^{d-1}\left|b_{j}^{\prime}\right|^{p}\right)^{\frac{q}{p}}\right) .
$$

To show (3.9), we observe that $\left|b_{j}^{\prime}\right| \geq\left|b_{j}\right|$, and thus it suffices to prove

$$
\left(\sum_{j=0}^{d-1}\left|b_{j}\right|^{p} e^{-p\left|b_{j}\right|}\right)^{\frac{1}{p}}-\left(\sum_{j=0}^{d-1}\left|b_{j}^{\prime}\right|^{p} e^{-p\|b\|_{\ell p}}\right)^{\frac{1}{p}} \geq 2^{-3} d^{-2}\left|b_{m^{*}}\right|^{2} .
$$

From the mean value theorem we obtain

$$
e^{-p\left|b_{j}\right|}-e^{-p\|b\|_{\ell p}} \geq p e^{-p\|b\|_{\ell p}}\left(\|b\|_{\ell^{p}}-\left|b_{j}\right|\right),
$$

and using $e^{-p\|b\|_{\ell p}} \geq e^{-p d\left|b_{m}\right|} \geq \frac{1}{2}$ we come to the inequality

$$
\left|b_{j}\right|^{p} e^{-p\left|b_{j}\right|}-\left|b_{j}^{\prime}\right|^{p} e^{-p\|b\|_{\ell^{p}}} \geq \frac{1}{2}\left|b_{j}\right|^{p}\left(p\|b\|_{\ell^{p}}-p\left|b_{j}\right|-\prod_{l \neq j}\left|a_{l}\right|^{p}+1\right) .
$$

Another application of the mean value theorem, this time for the function $t^{1 / p}$, gives

$$
p\|b\|_{\ell^{p}}-p\left|b_{j}\right| \geq d^{-1}\left|b_{m}\right|^{1-p}\left(\sum_{l \neq j}\left|b_{l}\right|^{p}\right) .
$$

On the other hand, we estimate:

$$
\begin{aligned}
& \prod_{l \neq j}\left|a_{l}\right|^{p}-1 \leq \prod_{l \neq j}\left|a_{l}\right|^{2}-1=\prod_{l \neq j}\left(1+\left|b_{l}\right|^{2}\right)-1 \leq e^{\sum_{l \neq j}\left|b_{l}\right|^{2}}-1 \\
& \leq e^{2^{-8} d^{-9}} \sum_{l \neq j}\left|b_{l}\right|^{2} \leq 2\left(\sum_{l \neq j}\left|b_{l}\right|^{p}\right)^{\frac{2}{p}} \leq 2 d\left|b_{m}\right|^{2-p}\left(\sum_{l \neq j}\left|b_{l}\right|^{p}\right),
\end{aligned}
$$


to conclude for every $j \neq m$ :

$$
p\|b\|_{\ell^{p}}-p\left|b_{j}\right|-\prod_{l \neq j}\left|a_{l}\right|^{p}+1 \geq 0,
$$

and for $j=m$ :

$$
p\|b\|_{\ell^{p}}-p\left|b_{m}\right|-\prod_{l \neq m}\left|a_{l}\right|^{p}+1 \geq 2^{-1} d^{-1}\left|b_{m}\right|^{1-p}\left|b_{m^{*}}\right|^{p} .
$$

Now by summing (3.11) over all $j=0, \ldots, d-1$ we get

$$
\sum_{j=0}^{d-1}\left|b_{j}\right|^{p} e^{-p\left|b_{j}\right|}-\sum_{j=0}^{d-1}\left|b_{j}^{\prime}\right|^{p} e^{-p\|b\|_{\ell p}} \geq 2^{-2} d^{-1}\left|b_{m}\right|\left|b_{m^{*}}\right|^{p}
$$

and then finally obtain (using the mean value theorem for $t^{1 / p}$ ):

$$
\begin{aligned}
& \left(\sum_{j=0}^{d-1}\left|b_{j}\right|^{p} e^{-p\left|b_{j}\right|}\right)^{\frac{1}{p}}-\left(\sum_{j=0}^{d-1}\left|b_{j}^{\prime}\right|^{p} e^{-p\|\|_{\ell} p}\right)^{\frac{1}{p}} \\
& \geq \frac{1}{p}\left(d\left|b_{m}\right|^{p}\right)^{\frac{1}{p}-1} 2^{-2} d^{-1}\left|b_{m}\right|\left|b_{m^{*}}\right|^{p} \geq 2^{-3} d^{-2}\left|b_{m}\right|^{2-p}\left|b_{m^{*}}\right|^{p} \geq 2^{-3} d^{-2}\left|b_{m^{*}}\right|^{2} .
\end{aligned}
$$

This is exactly (3.10), which completes the proof of Lemma 3.2.

Case 2. $\left|b_{m}\right|>t_{d}$, but $\left|b_{j}\right| \leq t_{d}$ for every $j \neq m$.

By (3.4) it is enough to prove

$$
\begin{aligned}
& (2 d)^{-5}\left(\frac{1}{d} \sum_{k=0}^{d-1}\left(1+\operatorname{arsinh}\left|B_{k}\right|\right)^{q / 2}\right)^{\frac{1}{q}} \\
& \leq\left((2 d)^{-5 p}\left(1+\operatorname{arsinh}\left|b_{m}\right|\right)^{p / 2}+\sum_{j \neq m}\left|b_{j}\right|^{p} e^{-p\left|b_{j}\right|}\right)^{\frac{1}{p}}
\end{aligned}
$$

and because $e^{-\left|b_{m^{*}}\right|} \geq e^{-t_{d}} \geq \frac{1}{2}$, it suffices to show

$$
\left(\frac{1}{d} \sum_{k=0}^{d-1}\left(1+\operatorname{arsinh}\left|B_{k}\right|\right)^{q / 2}\right)^{\frac{p}{q}} \leq\left(1+\operatorname{arsinh}\left|b_{m}\right|\right)^{p / 2}+2^{4 p} d^{5 p}\left|b_{m^{*}}\right|^{p}
$$




\section{Lemma 3.3.}

$$
\begin{aligned}
&\left(\frac{1}{d} \sum_{k=0}^{d-1}\left(1+\operatorname{arsinh}\left|B_{k}\right|\right)^{q / 2}\right)^{\frac{p}{q}} \leq\left(1+\operatorname{arsinh}\left(\frac{1}{d} \sum_{k=0}^{d-1}\left|B_{k}\right|^{q}\right)^{\frac{1}{q}}\right)^{\frac{p}{2}} \\
&\left(1+\operatorname{arsinh}\left(\frac{1}{d} \sum_{k=0}^{d-1}\left|B_{k}\right|^{q}\right)^{\frac{1}{q}}\right)^{\frac{p}{2}} \leq\left(1+\operatorname{arsinh}\left|b_{m}\right|\right)^{p / 2} \\
&+\frac{1}{\left|a_{m}\right|}\left(\left(\frac{1}{d} \sum_{k=0}^{d-1}\left|B_{k}\right|^{q}\right)^{\frac{1}{q}}-\left|b_{m}\right|\right) \\
&\left(\frac{1}{d} \sum_{k=0}^{d-1}\left|B_{k}\right|^{q}\right)^{\frac{1}{q}} \leq\left(\left|b_{m}\right|^{p}+\left|a_{m}\right|^{p} \sum_{j \neq m}\left|b_{j}\right|^{p}\right)^{\frac{1}{p}}+2^{4 p-1} d^{5 p}\left|a_{m}\right|\left|b_{m^{*}}\right|^{p} \\
&\left(\left|b_{m}\right|^{p}+\left|a_{m}\right|^{p} \sum_{j \neq m}\left|b_{j}\right|^{p}\right)^{\frac{1}{p}} \leq\left|b_{m}\right|+2^{4 p-1} d^{5 p}\left|a_{m}\right|\left|b_{m^{*}}\right|^{p}
\end{aligned}
$$

Estimate (3.12) follows by successively substituting left hand side of each inequality (3.14) -(3.16) into the preceding one. Also, we may assume $\left(\frac{1}{d} \sum_{k}\left|B_{k}\right|^{q}\right)^{1 / q}$ $\geq\left|b_{m}\right|$ in (3.14), since otherwise the desired estimate (3.12) trivially follows from (3.13).

Proof of Lemma 3.3. In order to prove (3.13), we consider the function

$$
\psi(t):=\left(1+\operatorname{arsinh}\left(t^{2 / q}\right)\right)^{\frac{q}{2}} .
$$

One can calculate:

$$
\begin{aligned}
& \psi^{\prime}(t)=t^{2 / q-1}\left(1+t^{4 / q}\right)^{-\frac{1}{2}}\left(1+\operatorname{arsinh}\left(t^{2 / q}\right)\right)^{\frac{q}{2}-1}, \\
& \psi^{\prime \prime}(t)=-\frac{1}{2 q} t^{2 / q-2}\left(1+t^{4 / q}\right)^{-\frac{3}{2}}\left(1+\operatorname{arsinh}\left(t^{2 / q}\right)\right)^{\frac{q}{2}-2} \\
& \cdot\left(2\left((q-2)+q t^{4 / q}\right) \operatorname{arsinh}\left(t^{2 / q}\right)+(q-2)\left(\left(1+t^{4 / q}\right)^{\frac{1}{2}}-t^{2 / q}\right)^{2}+(q-2)+4 t^{4 / q}\right),
\end{aligned}
$$

and conclude (using $q \geq 2$ ) that $\psi$ is increasing and concave on $[0, \infty)$. Jensen's inequality and elementary inequalities between power means (see [4]) give (3.13):

$$
\frac{1}{d} \sum_{k=0}^{d-1} \psi\left(\left|B_{k}\right|^{q / 2}\right) \leq \psi\left(\frac{1}{d} \sum_{k=0}^{d-1}\left|B_{k}\right|^{q / 2}\right) \leq \psi\left(\left(\frac{1}{d} \sum_{k=0}^{d-1}\left|B_{k}\right|^{q}\right)^{\frac{1}{2}}\right) .
$$

A couple of applications of the mean value theorem, for $(1+t)^{p / 2}$ and for arsinh $t$, together with $1 \leq p \leq 2, \sqrt{1+\left|b_{m}\right|^{2}}=\left|a_{m}\right|$, yield (3.14).

For (3.15) we first estimate the perturbation due to nonlinear terms:

$$
\begin{aligned}
\left|B_{k}-B_{k}^{\prime}\right| & \leq \sum_{j_{1}<j_{2}<j_{3}}\left|b_{j_{1}}\right|\left|b_{j_{2}}\right|\left|b_{j_{3}}\right|\left(\prod_{l \neq j_{1}, j_{2}, j_{3}}\left(\left|a_{l}\right|+\left|b_{l}\right|\right)\right) \\
& \leq d^{3}\left(1+2 t_{d}\right)^{d-3}\left(\left|a_{m}\right|+\left|b_{m}\right|\right)\left|b_{m^{*}}\right|^{2} \leq 4 d^{3}\left|a_{m}\right|\left|b_{m^{*}}\right|^{2}
\end{aligned}
$$


and furthermore compare:

$$
\begin{aligned}
\left|B_{k}^{\prime}-B_{k}^{\prime \prime}\right| & \leq\left|b_{m}\right|\left(\prod_{l \neq m}\left|a_{l}\right|-1\right)+\sum_{j \neq m}\left|a_{m}\right|\left|b_{j}\right|\left(\prod_{l \neq m, j}\left|a_{l}\right|-1\right) \\
& \leq d\left|a_{m}\right|\left(\prod_{l \neq m}\left|a_{l}\right|-1\right) \leq d\left|a_{m}\right|\left(e^{\frac{1}{2} \sum_{l \neq m}\left|b_{l}\right|^{2}}-1\right) \leq d^{2}\left|a_{m}\right|\left|b_{m^{*}}\right|^{2},
\end{aligned}
$$

where in the last line we used $e^{x}-1 \leq e^{x} x$ for $x \geq 0$. These two estimates can be combined, so that Minkowski's inequality, together with (3.6), $5 d^{3} \leq 2^{4 p-1} d^{5 p}$, and $\left|b_{m^{*}}\right|^{2} \leq\left|b_{m^{*}}\right|^{p}$, gives (3.15).

To deduce the last estimate (3.16), we use the mean value theorem for $t^{1 / p}$, and $\frac{\left|a_{m}\right|}{\left|b_{m}\right|} \leq 1+\frac{1}{\left|b_{m}\right|} \leq \frac{2}{t_{d}} \leq 2^{6} d^{5}$.

$$
\begin{aligned}
& \left(\left|b_{m}\right|^{p}+\left|a_{m}\right|^{p} \sum_{j \neq m}\left|b_{j}\right|^{p}\right)^{\frac{1}{p}}-\left|b_{m}\right| \leq\left(\left|b_{m}\right|^{p}\right)^{\frac{1}{p}-1}\left|a_{m}\right|^{p} \sum_{j \neq m}\left|b_{j}\right|^{p} \\
& \leq \frac{d\left|a_{m}\right|^{p}\left|b_{m^{*}}\right|^{p}}{\left|b_{m}\right|^{p-1}} \leq 2^{6 p-6} d^{5 p-4}\left|a_{m}\right|\left|b_{m^{*}}\right|^{p} \leq 2^{4 p-1} d^{5 p}\left|a_{m}\right|\left|b_{m^{*}}\right|^{p} .
\end{aligned}
$$

This proves Lemma 3.3 .

Case 3. $\left|b_{m}\right|>t_{d}$ and $\left|b_{m^{*}}\right|>t_{d}$.

Observe that it suffices to prove

$$
\beta_{d}\left(\left|B_{k}\right|\right)^{2} \leq \sum_{j=0}^{d-1} \beta_{d}\left(\left|b_{j}\right|\right)^{2}
$$

for every $k=0, \ldots, d-1$, because then by elementary inequalities for $\ell^{p}$ norms (see [4]) we have

$$
\left(\frac{1}{d} \sum_{k=0}^{d-1} \beta_{d}\left(\left|B_{k}\right|\right)^{q}\right)^{\frac{1}{q}} \leq \max _{0 \leq k \leq d-1} \beta_{d}\left(\left|B_{k}\right|\right) \leq\left(\sum_{j=0}^{d-1} \beta_{d}\left(\left|b_{j}\right|\right)^{2}\right)^{\frac{1}{2}} \leq\left(\sum_{j=0}^{d-1} \beta_{d}\left(\left|b_{j}\right|\right)^{p}\right)^{\frac{1}{p}} .
$$

The rest of the proof is a simple observation taken for instance from [5] or [8], but we include it for completeness. Split the set of indices $\{0, \ldots, d-1\}$ into

$$
J_{\text {big }}:=\left\{j:\left|b_{j}\right|>t_{d}\right\} \quad \text { and } \quad J_{\text {small }}:=\left\{j:\left|b_{j}\right| \leq t_{d}\right\},
$$

so in this case $\left|J_{\text {big }}\right| \geq 2$. Since the spectral norm of any $\left[\begin{array}{ll}a & \bar{b} \\ b & \bar{a}\end{array}\right] \in \mathrm{SU}(1,1)$ is equal to $|a|+|b|$, using submultiplicativity of operator norms and (2.2) we deduce

$$
\left|A_{k}\right|+\left|B_{k}\right| \leq \prod_{j=0}^{d-1}\left(\left|a_{j}\right|+\left|b_{j}\right|\right)
$$

which can, after taking logarithms, be written as

$$
\operatorname{arsinh}\left|B_{k}\right| \leq \sum_{j=0}^{d-1} \operatorname{arsinh}\left|b_{j}\right|
$$


By (3.4) one always has

$$
\beta_{d}\left(\left|B_{k}\right|\right)^{2} \leq(2 d)^{-10}\left(1+\operatorname{arsinh}\left|B_{k}\right|\right),
$$

and thus we estimate:

$$
\begin{aligned}
\beta_{d}\left(\left|B_{k}\right|\right)^{2} & \leq(2 d)^{-10}\left(1+\sum_{j \in J_{\mathrm{big}}} \operatorname{arsinh}\left|b_{j}\right|+\left|J_{\text {small }}\right| \operatorname{arsinh} t_{d}\right) \\
& \leq(2 d)^{-10} \sum_{j \in J_{\mathrm{big}}}\left(1+\operatorname{arsinh}\left|b_{j}\right|\right)=\sum_{j \in J_{\mathrm{big}}} \beta_{d}\left(\left|b_{j}\right|\right)^{2} \leq \sum_{j=0}^{d-1} \beta_{d}\left(\left|b_{j}\right|\right)^{2} .
\end{aligned}
$$

In the above calculation we used $d \operatorname{arsinh} t_{d} \leq d t_{d} \leq 1$ and $\left|J_{\text {big }}\right| \geq 2$.

This concludes the last case, and therefore Proposition 2.4 is established.

\section{A CLOSING REMARK}

While the estimate of Theorem 2.1 is independent of $p$, the proof makes it seriously dependent on $d$. It is not clear if the latter dependence can be avoided, but if so, it would require genuinely new methods. Suppose for a moment that we can construct $\beta=\beta_{d}$ as in Proposition 2.4 that does not depend on $d$. If we take $d \rightarrow \infty$ in (2.3), we will recover an analogue of Conjecture 1.1 on the group $\mathbb{Z}$ (as stated in [8]), and by an easy transference principle the actual Conjecture 1.1] (on $\mathbb{R}$ ) would also follow. Therefore, uniformization of the constants in $d$ turns out to be an even harder problem than the original one.

\section{REFERENCES}

1. M. Christ and A. Kiselev, Maximal functions associated to filtrations, J. Funct. Anal., 179 (2001), 409-425.

2. M. Christ and A. Kiselev, WKB asymptotic behavior of almost all generalized eigenfunctions for one-dimensional Schrödinger operators with slowly decaying potentials, J. Funct. Anal., 179 (2001), 426-447.

3. G. B. Folland, A course in abstract harmonic analysis, Stud. Adv. Math., CRC Press, Boca Raton, 1995.

4. D. S. Mitrinović and P. M. Vasić, Analytic inequalities, Springer-Verlag, New York, 1970.

5. C. Muscalu, T. Tao, and C. Thiele, A Carleson type theorem for a Cantor group model of the scattering transform, Nonlinearity 16 (2003), 219-246.

6. F. L. Nazarov and S. R. Treil, The hunt for a Bellman function: applications to estimates for singular integral operators and to other classical problems of harmonic analysis, Algebra i Analiz, Vol. 8 (1996), Issue 5, 32-162; English translation in St. Petersburg Math. J., 8 (1997), No. 5, 721-824.

7. R. Oberlin, A. Seeger, T. Tao, C. Thiele, and J. Wright, A variation norm Carleson theorem, to appear in J. Eur. Math. Soc.

8. T. Tao and C. Thiele, Nonlinear Fourier analysis, IAS/Park City Math. Institute, Graduate Summer School, 2003, Lecture Notes.

9. S. Verblunsky, On positive harmonic functions, Proc. London Math. Soc. 34 (1934), 125-157.

10. S. Verblunsky, On positive harmonic functions II, Proc. London Math. Soc. 40 (1935), 290320 .

Department of Mathematics, UCLA, Los Angeles, CA 90095-1555

E-mail address: vjekovac@math.ucla.edu 